\title{
IncRNA-ENST00000513396 is associated with multiple tumor signaling pathways, promotes the cell proliferation, invasion and migration in colorectal cancer
}

Jinxin Zhu

Nanjing Medical University

Hui Wang

Nanjing Medical University

Yangbin Du

Nanjing Medical University

Zhenyu He ( $\sim$ hezhenyu1968@163.com )

Nanjing Medical University

Research

Keywords: Colorectal cancer, Long non-coding RNA, Invasion, Bioinformatics

Posted Date: March 18th, 2021

DOl: https://doi.org/10.21203/rs.3.rs-309313/v1

License: (c) (i) This work is licensed under a Creative Commons Attribution 4.0 International License.

Read Full License 


\section{Abstract \\ Purpose}

Recently, many studies have revealed that Long noncoding RNAs (IncRNAs) were abundant in kinds of cells and might have multiple functions in a wide range of biological processes, such as proliferation, apoptosis, or cell migration. However, the role of IncRNA in the development of colorectal cancer has not been systematically reported and needs to be studied.

\section{Methods}

We applied Agilent microarray in tumoral tissues and paired para-cancer tissues for detecting different expression of IncRNAs and mRNAs. Bioinformatics analysis were used to identify the relationship between IncRNAs and corresponding mRNAs and the potential function of the IncRNAs. IncRNAENST00000430471 was chosen for further functional study through constructing overexpression plasmid, cell invasion, and cell migration.

\section{Results}

We found that 460 IncRNAs were significantly deregulated in cancer tissues. More importantly, they may participate in a variety of tumor-related signaling pathways through functional enrichment analysis with their co-expressed mRNAs, suggesting that these IncRNAs might play critical roles in tumorigenesis and development. Recently, we found IncRNA-ENST00000513396 was significantly upregulated in cancer tissues compared with para-cancer tissues. The overexpression of ENST00000513396 promotes tumor cell proliferation. Meanwhile ENST00000430471 could promote migration and invasion ability of colorectal cells.

\section{Conclusion}

LncRNAs play an important role in the development of colorectal cancer. Differentially expressed IncRNAs are associated with multiple tumor signaling pathways. Overexpression of IncRNA- ENST00000513396 in colorectal cancer cells can promote tumor cell proliferation and increase the ability of cell invasion and migration, which leads to the development of tumor.

\section{Introduction}

Colorectal cancer is the third most common malignant tumor in the world, with approximately 1.3 million new cases worldwide each year. ${ }^{1}$ In recent years, comprehensive treatment of colorectal cancer has made considerable progress, but its mortality rate is still increasing year by year; about 190, 000 patients per 
year died of colorectal cancer. ${ }^{2}$ The prognosis of colorectal cancer is closely related to the severity of the disease in diagnosis. Local or distant metastases often indicate a poor prognosis for patients. ${ }^{3}$ The occurrence and metastasis of tumor is a complex multi-step biological process. Epithelial-mesenchymal transition (EMT) is considered as a necessary condition for colorectal cancer metastasis, but the specific mechanism behind it is not yet clear. ${ }^{4}$ Therefore, in-depth discussion of the mechanism of colorectal cancer development and development, clarifying the key molecules and their roles in the process of colorectal cancer metastasis, is essential for early diagnosis and postoperative recurrence monitoring, which also helps to develop new drugs to block occurrence and development of colorectal cancer. Additionally, it is conducive to improving the survival rate of cancer patients and the quality of life of patients.

Long noncoding RNA (LnCRNA) is an RNA molecule with a sequence length greater than $200 \mathrm{nt}$. It does not encode a protein. The expression is spatiotemporal and tissue specific. ${ }^{5}$ It has various biological functions such as regulating cell proliferation, apoptosis, and metastasis. ${ }^{6-8}$ LncRNA can be regulated by chromosomal modification, histone modification, DNA methylation and other transcriptional regulation, and plays a regulatory role at the level of transcription and post-transcription. ${ }^{6,8-11}$ For example, LncRNA BCAR4 combined with Smad protein1 in the nucleus, restores the activity of histone acyltransferase p300, acetylates histone in the promoter region of glioma-associated oncogene 2 (GLI2), activates the Hedgehog/GLI2 transcription pathway, and promotes Breast cancer development. ${ }^{10}$ This shows that LncRNA plays an important role in the occurrence and development of tumors.

In recent years, high-throughput chip technology has become an important means of basic research. We detected the expression of IncRNA and mRNA in colorectal cancer tissues and adjacent tissues through the LncRNA chip technology. We found that there are 460 differentially expressed IncRNAs in colorectal cancer and adjacent tissues. Through functional enrichment analysis of these differentially expressed IncRNAs, it revealed that these genes are involved in a variety of tumor-related signaling pathways, suggesting that these differentially expressed IncRNAs play an important role in the development and metastasis of colorectal cancer. Through clinical sample testing, we have found that IncRNAENST00000513396 is significantly overexpressed in tumor tissues. Overexpression of ENST00000513396 can promote the proliferation of cell and improve the ability of invasion and migration of tumor cells.

This study helped us to explore the expression of IncRNA in colorectal cancer, found that some IncRNA may play an important role in the development and metastasis of colorectal cancer, and took ENST00000513396 as an example to explore its function and mechanism of action in order to find potential molecular markers and drug therapy targets to provide theoretical and experimental basis.

\section{Materials And Methods}

\section{Tissue samples}


The tumor tissues and corresponding adjacent tissues diagnosed by pathology in this research were all derived from the Second Affiliated Hospital of Nanjing Medical University. All patients signed an informed consent form.

The study protocol was approved by the ethics committee of the Second Affiliated Hospital of Nanjing Medical University.

\section{IncRNA gene chip and Bioinformatics analysis}

SurePrint G3 Human Gene Expression 8x60K v2 Microarray (Agilent, USA) was used to examine the expression of IncRNA and mRNA in 3 patients with colorectal cancer tumor tissues and adjacent tissues. Using Agilent Database Processing Software, Feature Extraction software (v11. 0.1. 1) obtained the data and chip map, and reading the value obtained the original data. GeneSpring GX v12. 1 software (Agilent, USA) was used to standardize and subsequently process the gene chip data. After normalizing the raw data, the high-quality screened probes were used for further analysis. Differentially expressed genes with statistical significance were obtained between the two groups of samples and filtrated by volcano graphs. Differentially expressed genes were filtrated by Fold Change. Hierarchical clustering was obtained through R script.

We used the Perl script to screen out protein coding genes adjacent to IncRNA (100Kb upstream and downstream) as cis-target genes of IncRNA. We predicted the target gene of IncRNA by calculating the Pearson correlation coefficient. When the absolute value of the Pearson correlation coefficient between the coding gene and IncRNA is bigger than 0.9 , we believed that the coding gene is the target gene of IncRNA. The standard enrichment calculation method was used for GO enrichment analysis and KEGG analysis.

By calculating the intersection of the coding gene collection co-expressed with IncRNA and the target gene collection of the transcription factor/chromatin regulatory complex, and using the hypergeometric distribution to calculate the enrichment degree of this intersection, the transcription factors significantly related to IncRNAs were obtained to predict the transcription factor/chromatin regulatory factor that possibly plays a regulatory role with IncRNAs. And a visual network diagram was drawn according to the results of hypergeometric distribution.

\section{Cell culture, plasmid construction and cell transfection}

The colorectal cancer cell lines (SW480) used in this experiment are all from the Institute of Biochemistry and Cell Research, Chinese Academy of Sciences (Shanghai). The medium of colorectal cancer cell line SW480 is a mixed medium of DMEM high glucose medium containing $10 \%$ fetal bovine serum.

We constructed the LncRNA overexpression plasmid. PCR amplified the G0138630-1 fragment and identify the primer sequence. The upstream primer was synthesized by General Bio: PEGFP-N-5 TGGGAGGTCTATATAAGCAGAG. Downstream primer: PCDNA3. 1R ACAGTGGGAGTGGCACCTTC. Positive clones were screened by bacterial solution PCR method. The obtained positive bacterial solution was 
shaken to extract plasmid at $37^{\circ} \mathrm{C}$ and sent to General Biological Company for sequencing. The constructed plasmid was transformed into SW480 cells for targeted-gene expression detection.We transfected siRNA into sw480cells by lipo2000 transfection reagent and performed the next QPCR detection. The siRNA is shown in Table 1.

Table 1

The siRNA design for the knockdown of IncRNA (The first is the forward primer and the second is the reverse primer)

\begin{tabular}{|c|c|}
\hline Primers & Primer sequences \\
\hline \multirow[t]{2}{*}{ Control } & CAAGATCATGGCAGGCATCC \\
\hline & TCTCAACAGCTССААССТСА \\
\hline \multirow[t]{2}{*}{ NC } & UUACUUUUCCCUCAUUUGGUU \\
\hline & CCGAAUAAGGGAUAAGAAAGG \\
\hline \multirow[t]{2}{*}{ SiRNA1 } & UUUUACUUCCCUUUCAUGGUU \\
\hline & CCAUGAAAGGGAAGUAAAAGG \\
\hline \multirow[t]{2}{*}{ SiRNA2 } & UCAGAUUUCUCAUUGAGUCCA \\
\hline & GACUCAAUGAGAAAUCUGAAG \\
\hline \multirow[t]{2}{*}{ SiRNA3 } & AGAACUUUGGUGUCAUUGGUC \\
\hline & CCAAUGACACCAAAGUUCUGA \\
\hline
\end{tabular}

\section{Real-time PCR Assay}

Total RNA was extracted from CRC cells using the Trizol method (Synthgene, China). Reverse transcription was performed using reverse transcriptase kit (Synthgene, China) according to the manufacturer's protocol.

Real-time PCR was performed using an SYBR premix Ex Taq kit on 12000 Real-Time PCR System (Applied Biosystems, USA) according to the manufacturer's protocol.

The primer sequences used were obtained from General Bio.

\section{Cell proliferation, invasion, and migration assay}

Collected the logarithmic phase cells, treated with MTT(Synthgene, China) and dimethyl sulfoxide, and then measured the absorbance of each well at OD490nm of the enzyme-linked immunoassay detector(hermo scientific, USA).

The transwell inserts were firstly treated with Matrigel $(60 \mu \mathrm{l})$. Then, HCT116 cells transfected with GAPLINC siRNAs or control siRNAs were collected and resuspended in serum-free DMEM medium. Subsequently, the cell suspensions was added into the upper chamber of the inserts, and the complete 
medium (containing 20\% FBS) were added into the lower chamber of the transwell. Finally, a microscope was applied to take images of the invaded cells after the cells were stained with $0.1 \%$ crystal violet.

Cells were seeded and transfected on six-well plates with targeted siRNA or control, then an artificial scratch wound on a confluent monolayer of the cells was created with a 200ul pipette tip. Observe the width of the track in different treatment groups after taking the scratch for 0 hours and 24 hours. Each experiment was repeated three times..

\section{Statistical Analysis}

Data were expressed as the mean \pm SD of at least three independent experiments. Statistical analysis was carried out using The SPSS 21.0 software package (SPSS, Chicago, IL). Student's t-test or ANOVA was performed to analyze the data. $\mathrm{P}<0.05$ was considered statistically significant.

\section{Results}

\section{Differentially expressed LncRNAs in colorectal cancer and adjacent tissues}

We randomly selected 3 cases of colorectal cancer tumor tissue and relative para-cancerous tissues, extracted total RNA, and detected the expression profile of IncRNA using the Agilent IncRNA chip. It was found that 460 IncRNAs (change factor $\geq 2, P \leq 0.05$, calculated by student $t$ test), of which 326 IncRNAs were highly expressed and 134 IncRNAs were lowly expressed (Fig. 1).

Cis-acting target gene prediction means that IncRNA affects its spatially adjacent protein-coding genes through related cis-acting elements, thereby regulating gene expression. The IncRNA target gene is predicted based on the positional relationship between IncRNA and gene. Trans-target gene prediction refers to the case where there is a positive or negative correlation between the expression of LncRNA and some distant genes, and the target gene can be predicted by analyzing the correlation between the expression of IncRNA and protein-coding genes between samples. We used the Perl script to screen out protein coding genes adjacent to IncRNA (100Kb upstream and downstream) as cis-target genes of IncRNA. By calculating the Pearson correlation coefficient between the coding gene and IncRNA to predict the target gene of IncRNA, when the absolute value of the Pearson correlation coefficient between the two is greater than 0.9 , we think that the coding gene is the target gene of IncRNA (Table 2 and tableS1). 
Table 2

LncRNA target gene prediction results based on positional relationship (partial)

\begin{tabular}{|ll|}
\hline \#IncRNA & Genes \\
\hline ABCC6P1 & NOMO2 \\
\hline ABCC6P2 & NOMO1; NPIPA3; NPIPA2 \\
\hline ABHD11 & ABHD11; STX1A; WBSCR22; WBSCR27; VPS37D; CLDN4; CLDN3; DNAJC30 \\
\hline AC000079.1 & CDC45; UFD1L; HIRA; C22Orf39; MRPL40 \\
\hline AC002117.1 & NMT1; HEXIM2; CAKD; ACBD4; PLCD3; FMNL1; SPATA32;HEXIM1 \\
\hline AC005682.8 & TOMM7;FAM126A \\
\hline
\end{tabular}

We also did GO enrichment analysis and KEGG enrichment analysis of differentially expressed IncRNA cis-target gene and trans-target gene (Fig. 2 and Fig. 3). We have found some signal molecules or pathways related to tumors, such as cell adhesion molecules(CAMs), transcriptional misregulation in cancer, and Rap 1 signaling pathway.Based on existing research, IncRNA interacts with transcription factors to affect downstream gene expression. Through association analysis of IncRNA-transcription factor, we found that ACAP1, A4AGLT, and ABCF2 were related to multiple IncRNAs, such as ENST00000513396, while ACAP1, A4AGLT, and ABCF2 were all related to the occurrence and development of tumors, which suggests that the interaction between IncRNA and transcription factors may play an important role in the process of colorectal cancer metastasis (Fig. 4).

\section{Cell biological behaviors in vitro after Overexpression of ENST00000513396}

We selected 10 IncRNAs with significantly different expressions and verified them in clinical tissue samples. It was found that the expression difference of IncRNA-ENST00000513396 was the most obvious in tumor tissues, so we chosen ENST00000513396 for the next experiment. We conducted indepth research through constructing ENST00000513396 to knockout expressed and overexpressed cell lines (Fig. 5).The results are as follows.

Firstly, we observed the effect of overexpression of ENST00000513396 on cell proliferation and other biological functions by MTT method. We found that overexpression of ENST00000513396 can significantly enhance the proliferation ability of colon cancer cell line HCT116, suggesting that overexpression of ENST00000513396 can enhance the proliferation ability of tumor cells in vitro (Fig. 6).

Secondly, we analyzed the effect of ENST00000513396 on the migration ability of colon cancer cells using a cell scratch test. The results showed that after overexpression of ENST00000513396, the migration ability of tumor cells was significantly improved (Fig. 7). 
And finally, we used Transwell cell technology to analyze the effect of ENST00000513396 on the invasion ability of colon cancer cells. The results showed that after overexpression of ENST00000513396, the invasion ability of colon cancer cells was significantly improved (Fig. 8).

So in summary, the overexpression of ENST00000513396 can obviously promote the proliferation, migration and invasion of colon cancer cells in vitro.

\section{Discussion}

Colorectal cancer (CRC) is one of the common malignant tumors worldwide. Despite the tremendous achievements made in surgery, chemotherapy, and biotherapy in recent years, about 700, 000 patients still die from colorectal cancer each year, due to the lack of early diagnosis and tumor metastasis and recurrence. ${ }^{12}$ Therefore, in-depth research on the mechanism of the occurrence and development of colorectal cancer is expected to make a breakthrough in the early diagnosis, metastasis and targeted therapy of tumors.

Non-coding RNA plays a broad and powerful regulatory role in cells. In recent years, with the progress of research, it has been confirmed that it plays an important role in the process of tumorigenesis and development, and becomes a hot spot in recent years. ${ }^{13,14}$ RNA that exceeds 200 nt in length is called long noncoding RNA (IncRNA). LncRNA has obvious spatial and temporal specificity and tissue specificity. Previous reports have shown that IncRNA plays an important role in many physiological processes such as cell proliferation and differentiation. ${ }^{15-17}$ In recent years, with the deepening of the research on the mechanism of tumorigenesis and development, the abnormal expression of IncRNA in various tumors has been discovered, indicating that it plays an important role in the process of tumorigenesis and development, and may provide new methods for the diagnosis and treatment of colorectal cancer. ${ }^{18-20}$

In this study, through high-throughput chip technology, combined with bioinformatics analysis methods, to find differentially expressed IncRNA in colorectal cancer and adjacent tissues, we found 460 differentially expressed IncRNA (change factor $\geq 2, p \leq 0.05$ ). Through functional enrichment analysis of the co-expressed mRNA, it was found that the co-expressed mRNA function of these differentially expressed IncRNAs was mostly related to tumor-related signaling pathways, indicating that these differentially expressed IncRNAs may play an important role in the occurrence and development of colorectal cancer.

We selected IncRNA-ENST00000513396 with obvious differential expression as the research object. Through functional enrichment of its co-expressed mRNA, it was found to be related to multiple tumors signaling pathways. At the same time, we verified in clinical samples and found that it is the highest expressed in tumor tissues, so we determined ENST00000513396 as the research object to further study its function and mechanism of action. We constructed the overexpression and knockout plasmids of ENST00000513396. in vitro experiments, it was found that after overexpression of ENST00000513396, 
the proliferation of cells was accelerated, and the proliferation rate of colorectal cancer cells that knocked down the expression of ENST00000513396 decreased, suggesting that overexpression of

ENST00000513396 can promote tumor proliferation in vitro. Cell scratch experiments and Transwell cell experiments showed that overexpression of ENST00000513396 can improve the ability of tumor cells to migrate and invade, which is closely related to the occurrence and development of tumors. In summary, ENST00000513396 can promote the proliferation of tumor cells and increase the ability of tumor cells to invade and migrate. Therefore, ENST00000513396 may play an important role in the development of colorectal cancer.

Although this subject studied the function of ENST00000513396 in vitro, it has not been verified in vivo and the relationship between it and the prognosis of clinical patients has not been studied, which needs further improvement. It is essential to continue to explore the key molecules and signaling pathways that affect the ability of colorectal cancer to proliferate, migrate and invade, and work hard to study new diagnostic and therapeutic methods. Therefore, this will be the direction of our further discussion.

This subject systematically analyzes the abnormal expression of IncRNA in colorectal cancer tissues and adjacent tissues, and takes ENST00000513396 as the research object to elaborate its impact on cell function, providing a theory for its use as a diagnostic marker and therapeutic target for colorectal cancer basis.

\section{Conclusion}

In this study, IncRNA chips were used to analyze colorectal cancer tissues and adjacent tissues, and 460 differentially expressed IncRNAs were screened. Combined with bioinformatics analysis, these differentially expressed IncRNAs were found to be related to multiple tumor signaling pathways.

LncRNA-ENST00000513396 is highly expressed in colorectal cancer tissues. The results of in vitro experiments show that ENST00000513396 high expression promotes tumor cell proliferation, and can increase the ability of tumor cell migration and invasion.

These fundings contribute to a better understanding of the potential mechanism of the progression of $\mathrm{CRC}$, which could be a novel diagnostic and therapeutic target for CRC.

\section{Declarations}

\section{Ethics approval and consent to participate}

This study was approved by the Ethics Committee of the The Second Affiliated Hospital of Nanjing Medical University ([2018]KY No.25). Informed consent was obtained from all participants included in the study and experimental procedures were performed according to the guidelines of the non-profit, statecontrolled HTCR (Human Tissue and Cell Research) foundation. 


\section{Consent for publication}

Not applicable.

\section{Availability of data and materials}

Datasets are available on request from the corresponding author on reasonable request. The raw data and all related documents supporting the conclusions of this manuscript will be made available by the authors, without undue reservation, to any qualified researcher.

\section{Funding and acknowledgements}

This research was supported by the Nanjing Science and Technology Development Plan Project(2018sc512012).

\section{Author Contributions}

All authors contributed toward data analysis, drafting, and revising the paper and agree to be accountable for all aspects of the work.

\section{Competing interests}

The authors report no conflicts of interest in this work.

\section{Author details}

Jinxin zhu. E-mail: 737419628@qq.com

Hui Wang. E-mail:359718219@qq.com

Yangbin Du. E-mail:1760661092@qq.com

Zhenyu He. E-mail:hezhenyu1968@163.com

\section{References}

1. Siegel, R. , J. Ma, Z. Zou, and A. Jemal, Cancer statistics, 2014. CA Cancer J Clin, 2014. 64(1): p. 9-29.

2. Chen, W. , et al. , Cancer statistics in China, 2015. CA Cancer J Clin, 2016. 66(2):p. 115-32.

3. Wang, W. , Y. Li, X. Zhang, J. Jing, X. Zhao, Y. Wang, and C. Han, Evaluating the significance of expression of CEA mRNA and levels of CEA and its related proteins in colorectal cancer patients. $J$ Surg Oncol, 2014. 109(5): p. 440-4.

4. A. Lopez-Soto., et al. , Control of metastasis by NK cells. Cancer Cell, vol. 32, no. 2, pp. 135-154, 2017. 
5. Derrien, T. , R. Johnson, G. Bussotti, A. Tanzer, S. Djebali, H. Tilgner, et al, The GENCODE v7 catalog of human long noncoding RNAs: analysis of their gene structure, evolution, and expression. Genome Res, 2012. 22(9): p. 1775-89.

6. Yuan, J. H. , F. Yang, F. Wang, J. Z. Ma, Y. J. Guo, Q. F. Tao and et al. A long noncoding RNA activated by TGF-beta promotes the invasion-metastasis cascade in hepatocellular carcinoma. Cancer Cell, 2014. 25(5): p. 666-81.

7. Hu, X. , Y. Feng, D. Zhang, S. D. Zhao, Z. Hu, J. Greshock and et al, A functional genomic approach identifies FAL1 as an oncogenic long noncoding RNA that associates with BMI1 and represses p21 expression in cancer. Cancer Cell, 2014. 26(3): p. 344-57.

8. Bao, X. , H. Wu, X. Zhu, X. Guo, A. P. Hutchins, Z. Luo and et al. The p53-induced lincRNA-p21 derails somatic cell reprogramming by sustaining $\mathrm{H} 3 \mathrm{~K} 9 \mathrm{me} 3$ and $\mathrm{CpG}$ methylation at pluripotency gene promoters. Cell Res, 2015. 25(1): p. 80-92.

9. DeOcesano-Pereira, C. , M. S. Amaral, K. S. Parreira, A. C. Ayupe, J. F. Jacysyn, G. P. AmaranteMendes, E. M. Reis and et al, Long non-coding RNA INXS is a critical mediator of BCL-XS induced apoptosis. Nucleic Acids Res, 2014. 42(13): p. 8343-55.

10. Xing, Z. , A. Lin, C. Li, K. Liang, S. Wang, Y. Liu, P. K. Park and et al. IncRNA directs cooperative epigenetic regulation downstream of chemokine signals. Cell, 2014. 159(5): p. 1110-25.

11. Arab, K. , Y. J. Park, A. M. Lindroth, A. Schafer, C. Oakes, D. Weichenhan, A. Lukanova, E. Lundin and et al. Long noncoding RNA TARID directs demethylation and activation of the tumor suppressor TCF21 via GADD45A. Mol Cell, 2014. 55(4): p. 604-14.

12. Ferlay, J. , I. Soerjomataram, R. Dikshit, S. Eser, C. Mathers, M. Rebelo, D. M. Parkin and et al, Cancer incidence and mortality worldwide: sources, methods and major patterns in GLOBOCAN 2012. Int J Cancer, 2015. 136(5): p. E359-86.

13. Carninci, P. , T. Kasukawa, S. Katayama, J. Gough, M. C. Frith, N. Maeda, R. Oyama and et al. Genome Science, The transcriptional landscape of the mammalian genome. Science, 2005. 309(5740): p. 1559-63.

14. Djebali, S. , C. A. Davis, A. Merkel, A. Dobin, T. Lassmann, A. Mortazavi and et al. Landscape of transcription in human cells. Nature, 2012. 489(7414): p. 101-8.

15. Guan, D. , W. Zhang, W. Zhang, G. H. Liu, J. C. Belmonte, Switching cell fate, ncRNAs coming to play. Cell Death Dis, 2013. 4: p. e464.

16. Lee, J. T. , M. S. Bartolomei, X-inactivation, imprinting, and long noncoding RNAs in health and disease. Cell, 2013. 152(6): p. 1308-23.

17. Kung, J. T. , D. Colognori, J. T. Lee, Long noncoding RNAs: past, present, and future. Genetics, 2013. 193(3): p. 651-69.

18. Renganathan A, Felley-Bosco E. Long Noncoding RNAs in Cancer and Therapeutic Potential. Adv Exp Med Biol. 2017;1008:199-222.

19. Wang J, Su Z, Lu S, Fu W, Liu Z, Jiang X, Tai S. LncRNA HOXA-AS2 and its molecular mechanisms in human cancer. Clin Chim Acta. 2018 Oct;485:229-233. 
20. Chandra Gupta S, Nandan Tripathi Y. Potential of long non-coding RNAs in cancer patients: From biomarkers to therapeutic targets. Int J Cancer. 2017 May 1;140(9):1955-1967.

\section{Figures}

A

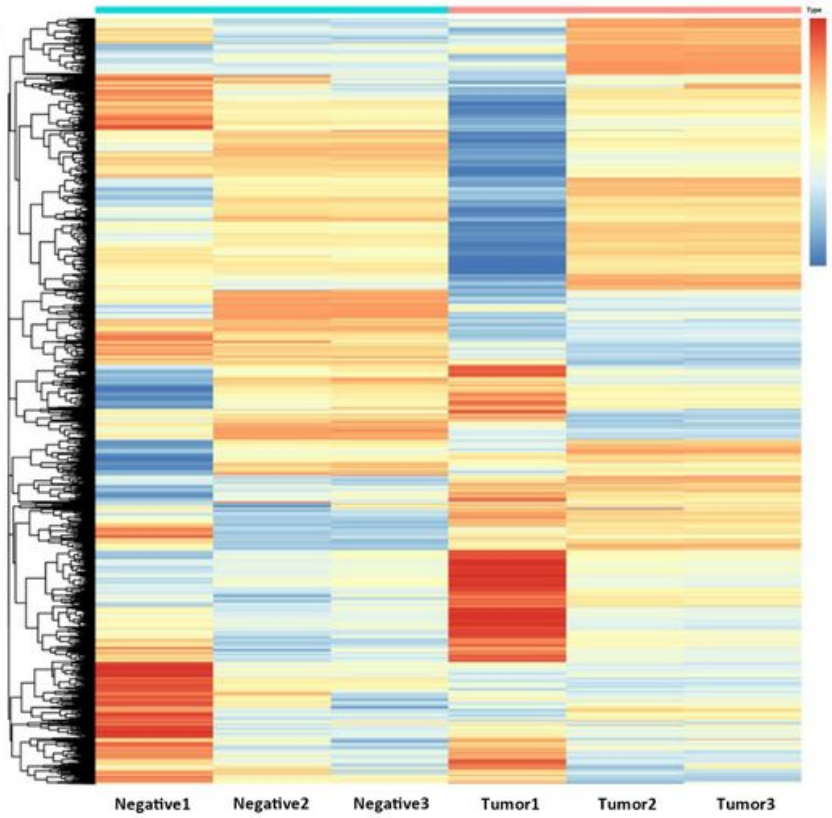

C

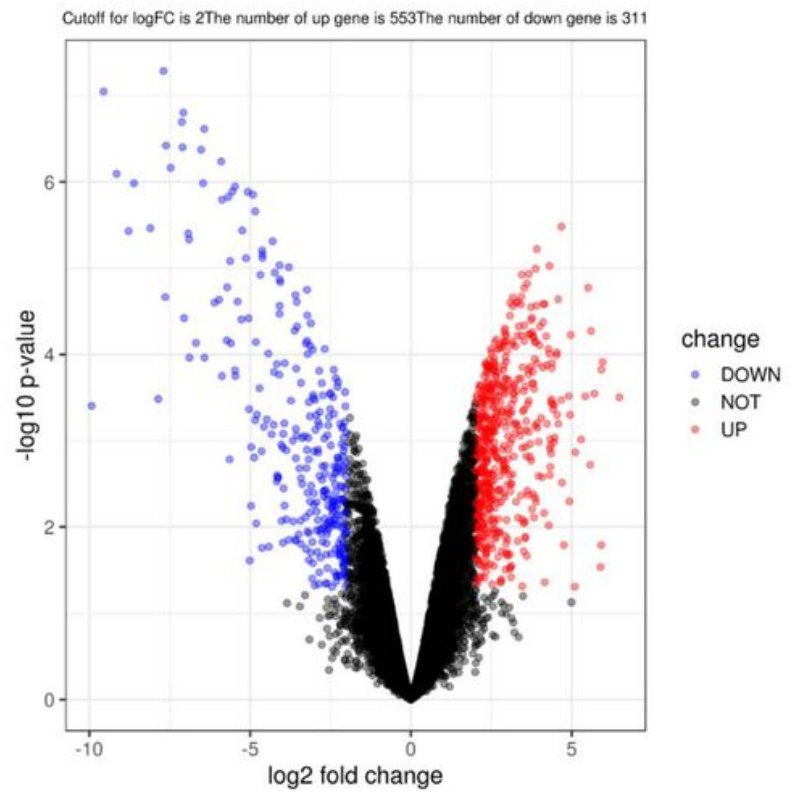

B

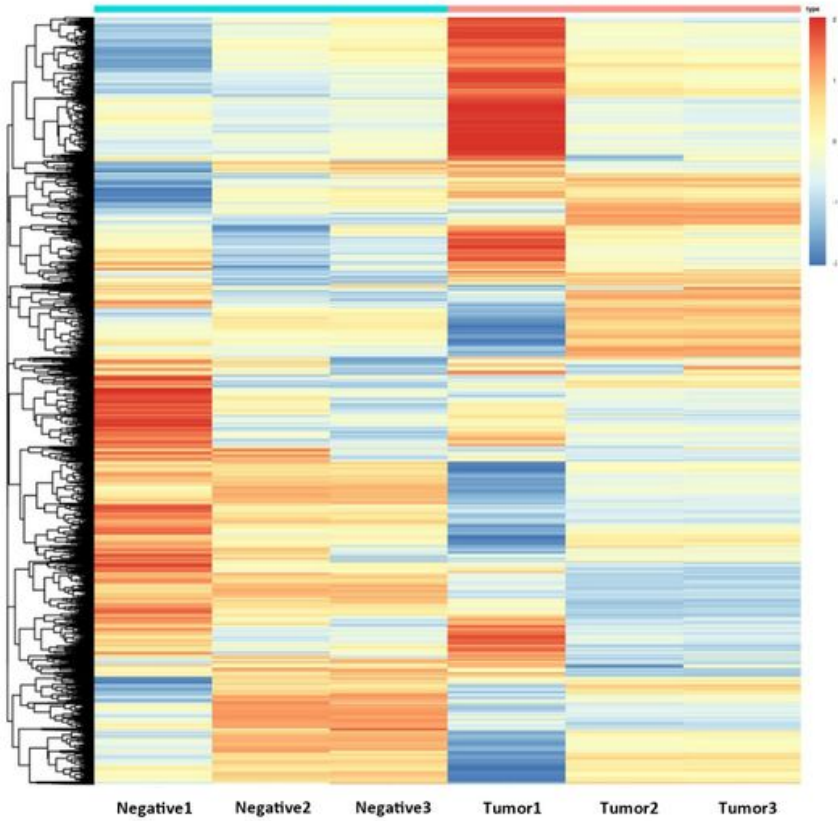

D

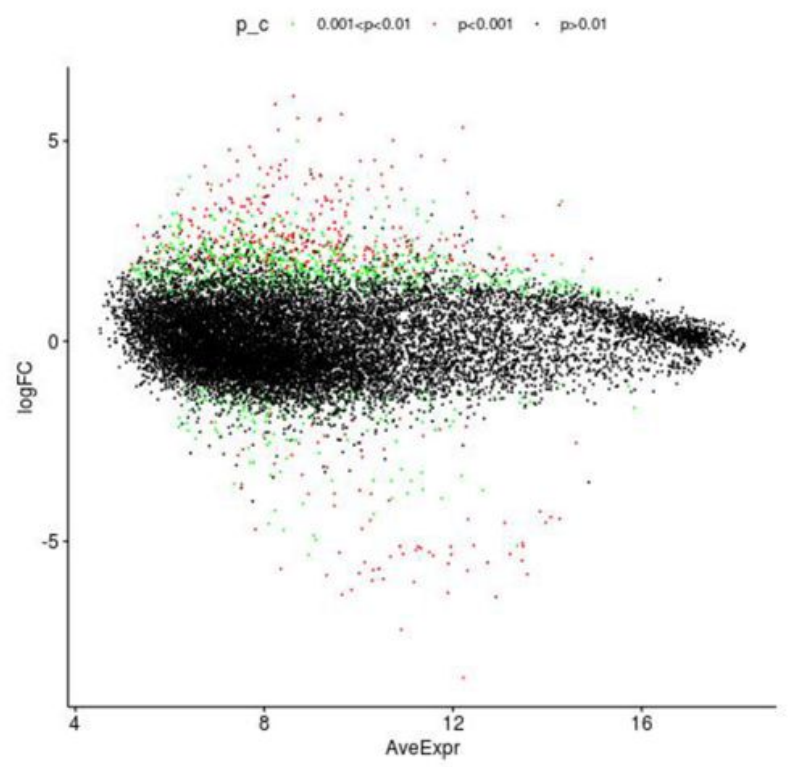

Figure 1

Differentially expressed LncRNA in colorectal cancer and adjacent tissues. A: Hierarchical cluster analysis of differentially expressed mRNA. B: Differential expression IncRNA hierarchical cluster analysis. C: Volcano graph of differentially expressed IncRNA. The abscissa is log2FC and the ordinate is $\log 10$ 
(corrected $p$-value). The red dots are up-regulated genes. The blue dots are down-regulated genes. The gray dots are not significant genes. D: MA map of differentially expressed IncRNA. The X-axis is the average value of all samples for comparison after normalization. The Y-axis is log2 (FoldChange). Significant (according to differential screening conditions) differential genes are marked in red.

A

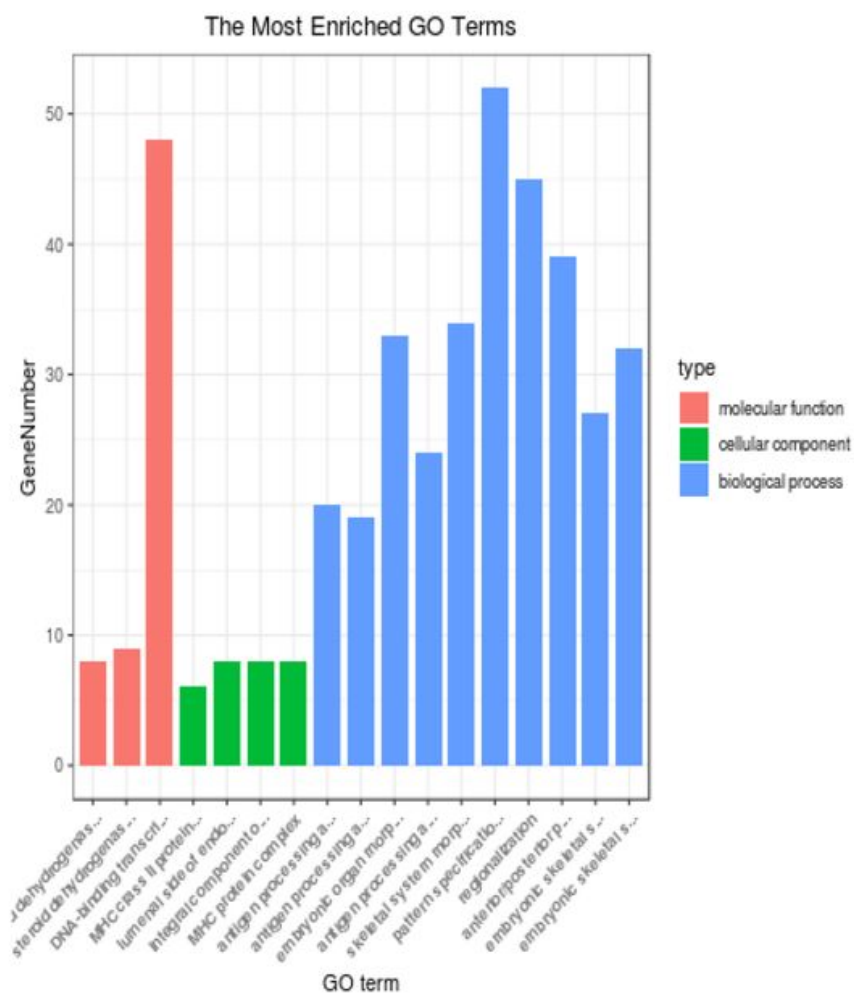

B

EnrichmentKEGG

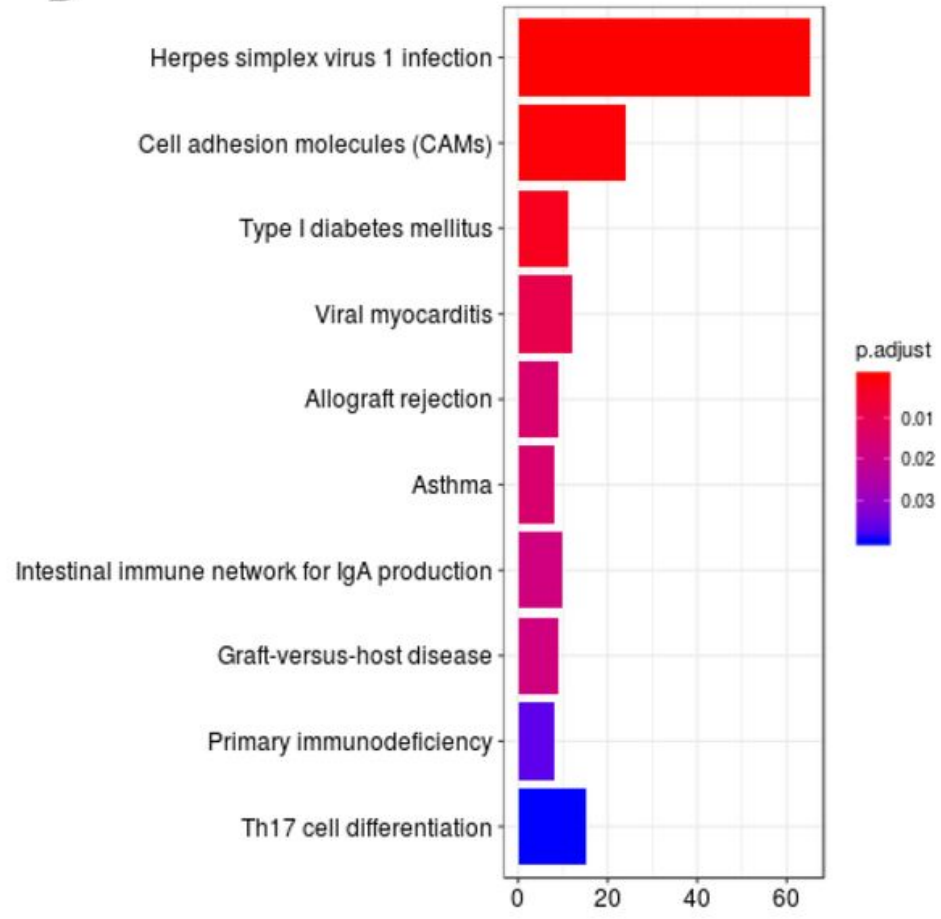

Figure 2

Enrichment analysis of IncRNA cis-target genes with differential expression. A is GO enrichment analysis (abscissa is count: the number of genes enriched to the entry; ordinate Terms: the entry enriched by GO, including GO description, the red color means the smaller the p-value, the more obvious the enrichment. Horizontal The axis is GeneRatio, which represents the ratio of the number of differential genes under the $\mathrm{GO}$ term to the total number of differential genes, and the ordinate is the enrichment entries. $B$ is the KEGG enrichment analysis (the abscissa is the count, and the ordinate is the number of enrichment). 
A

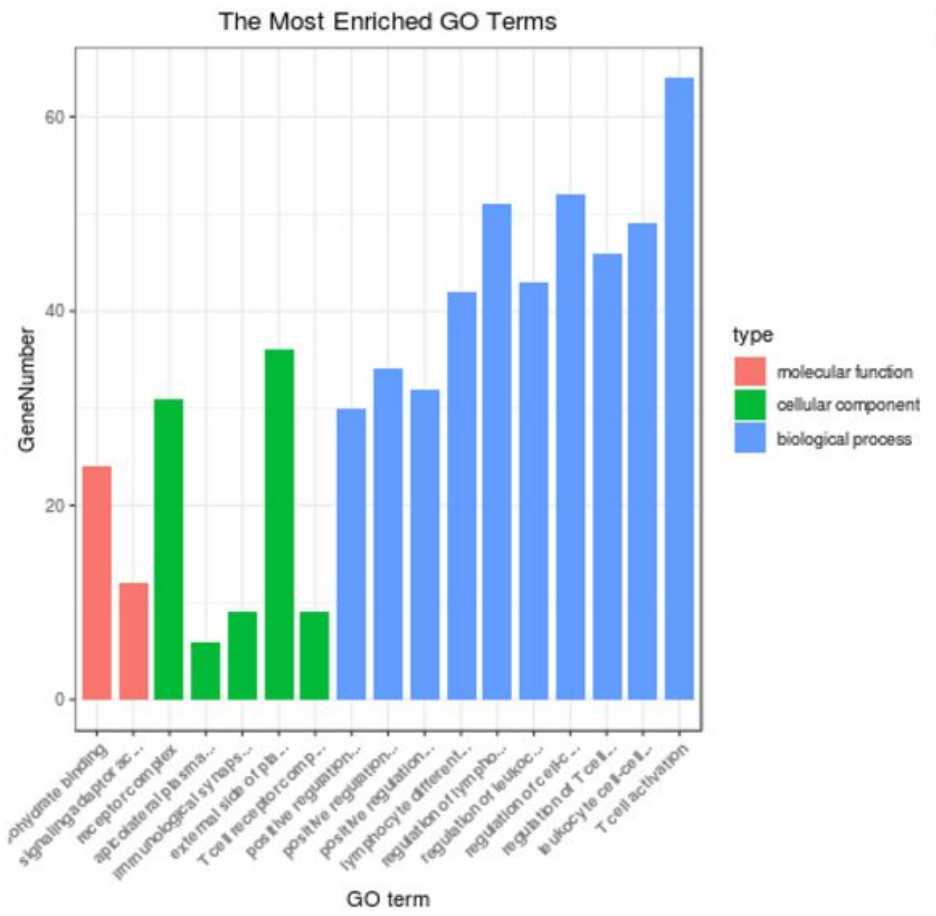

B

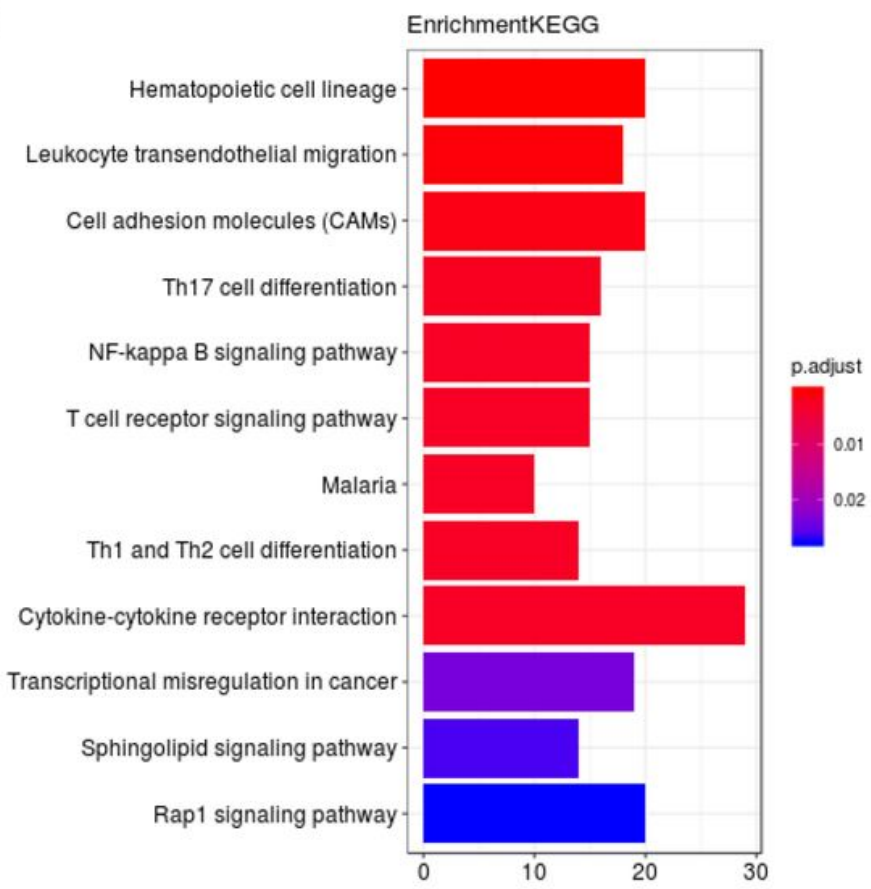

Figure 3

Enrichment analysis of IncRNA trans-target genes with differential expression. A is GO enrichment analysis (abscissa is count: the number of genes enriched to the entry; ordinate Terms: entries enriched by $\mathrm{GO}$, including $\mathrm{GO}$ description, the red color means the smaller the $\mathrm{p}$-value, the more obvious the enrichment). $B$ is KEGG enrichment analysis (abscissa is count, ordinate is enrichment number). 


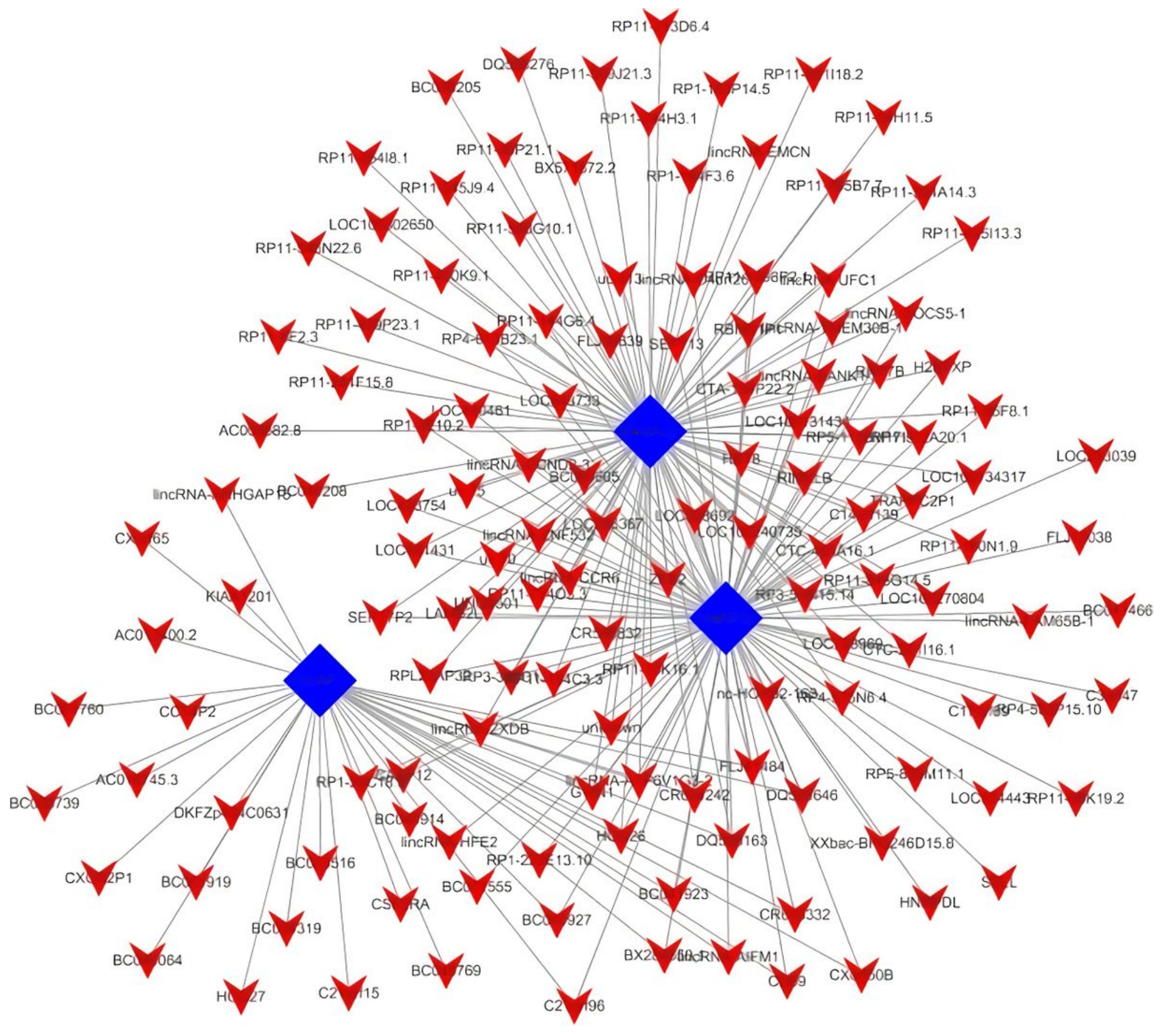

Figure 4

IncRNA-transcription factor regulatory network. Note:Red represents IncRNA and blue represents transcription factor. 


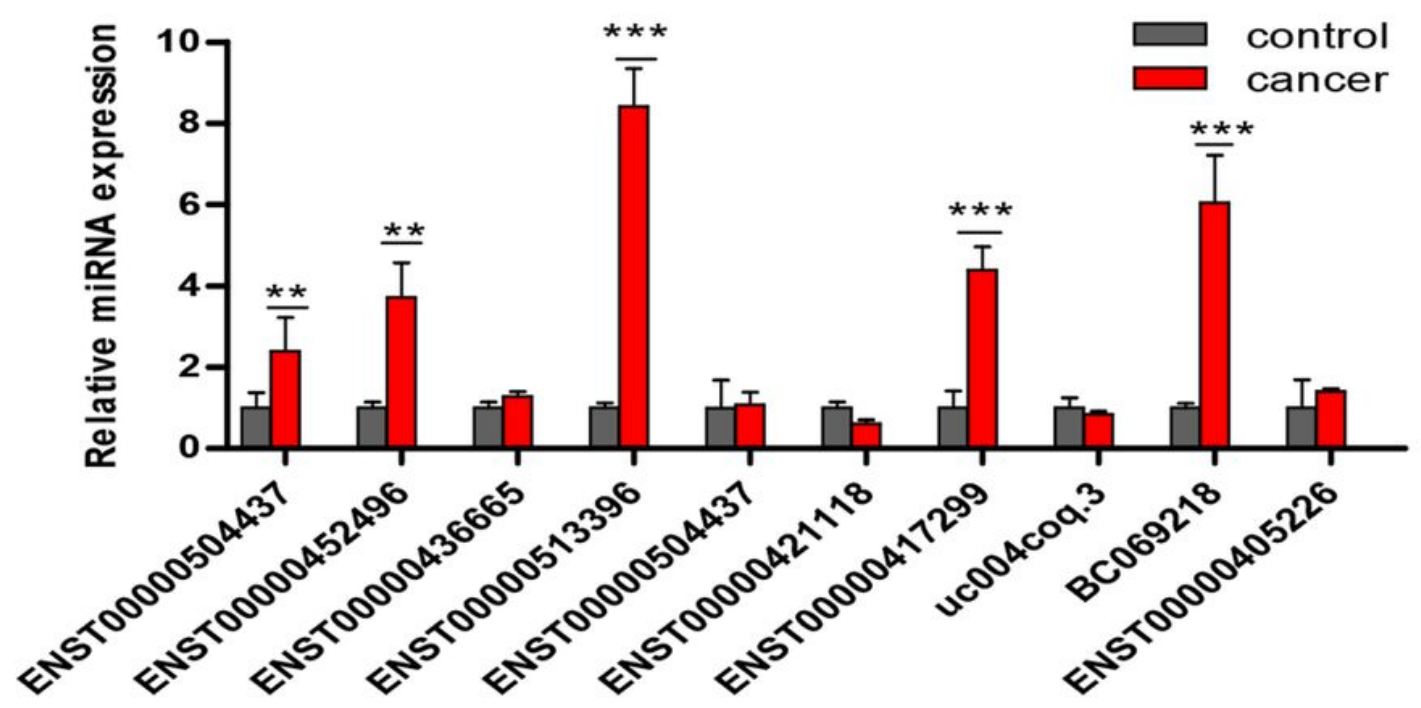

B

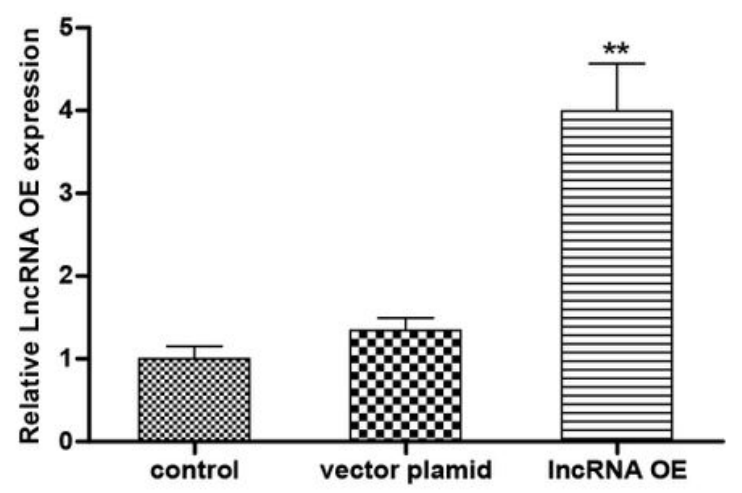

$\mathrm{C}$

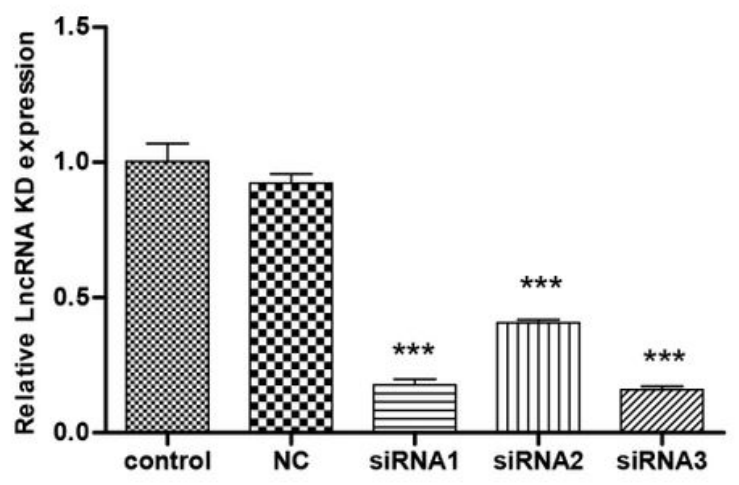

Figure 5

ENST00000513396 was verified in clinical specimens and cell lines and constructed overexpression plasmids. A: The expression of IncRNA in clinical samples, of which ENST00000513396 has the most obvious difference in clinical specimens. B: Expression of ENST00000513396 after SW480 cell line was transfected with empty and overexpression plasmids. C: Expression of ENST00000513396 after SW480 cell line was transfected with empty and knockout expression plasmids, respectively. 


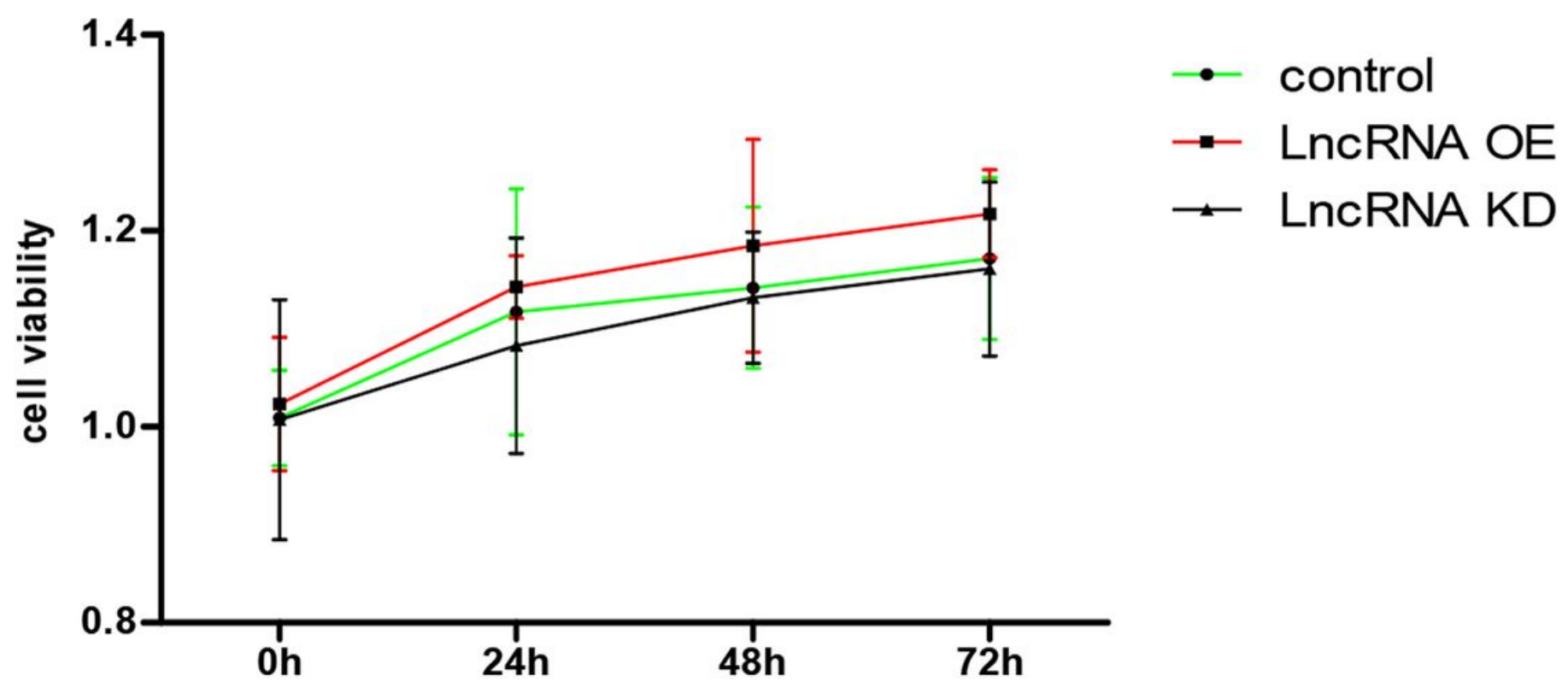

Figure 6

The effect of overexpression of ENST00000430471 on tumor proliferation in vitro. MTT test results of the proliferation ability of tumor cell line SW480 after overexpression of ENST00000513396; the proliferation ability of tumor cell line SW480 was weakened after ENST00000513396 was knocked out. ${ }^{*}<<0.05$, calculated by student $\mathrm{t}$ test. 
A

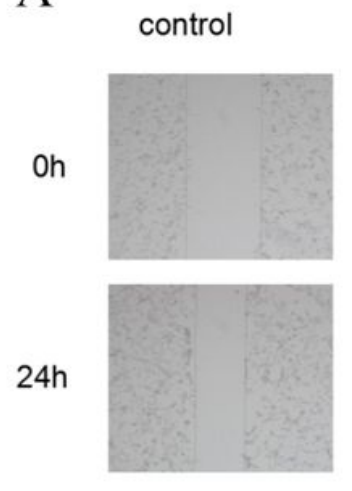

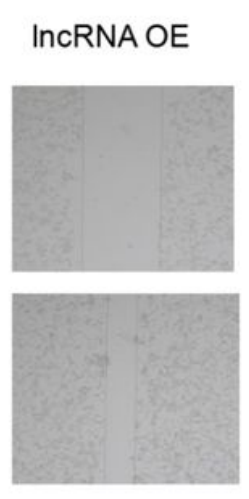
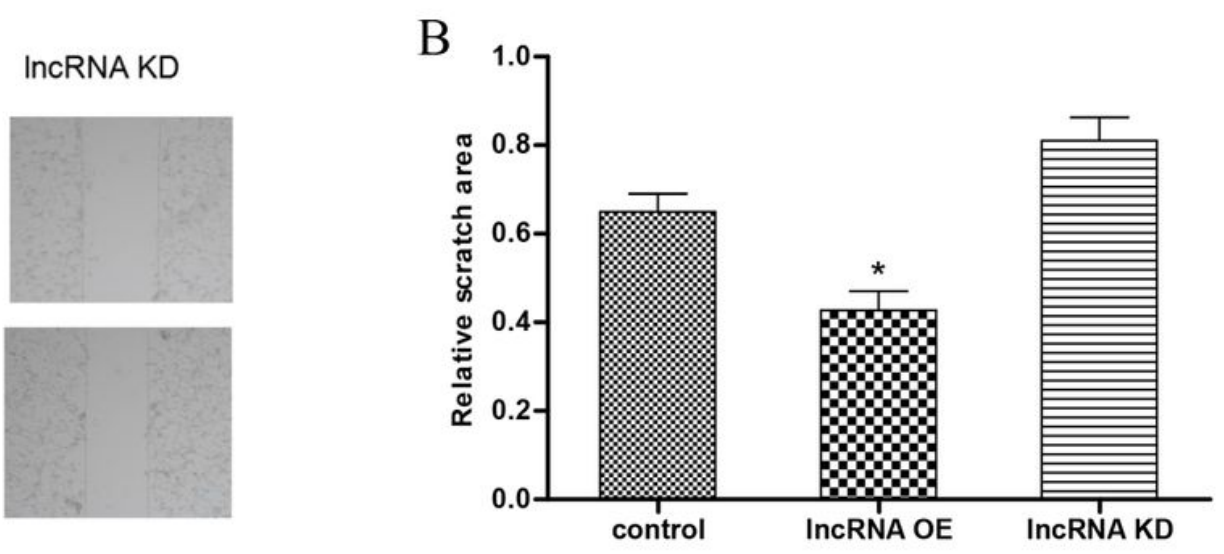

\section{Figure 7}

Effect of overexpression of ENST00000513396 on colon cancer cell migration ability. A: Test result of scratch test of tumor cell line SW480 migration ability after overexpression of ENST00000513396; B: area of scratch area. ${ }^{*} \mathrm{P}<0.05$, calculated by student $t$ test.

A

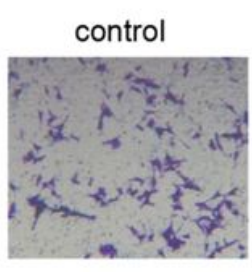

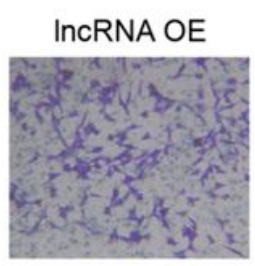

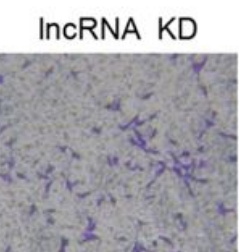

$\mathrm{B}$

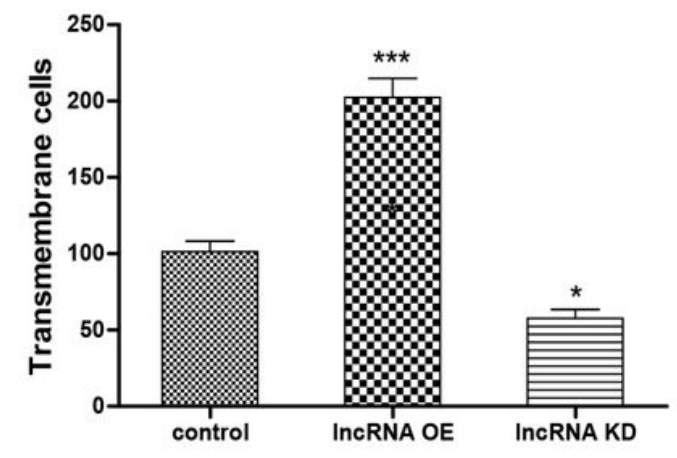

\section{Figure 8}

The effect of overexpression of ENST00000513396 on the invasion and migration ability of colon cancer cells. A: Transwell chamber test results of tumor cell line SW480 invasion ability after overexpression of ENST00000513396; B: Data statistics of invading tumor cells. ${ }^{*} P<0.05,{ }^{\star \star *} P<0.001$, calculated by student t test.

\section{Supplementary Files}

This is a list of supplementary files associated with this preprint. Click to download. 
- TableS1.docx

Page 19/19 